\title{
Gastric Foreign Bodies in Prison Inmates
}

\author{
Manish Kumar ${ }^{1}$ Sanjeev Sachdeva ${ }^{1}$ Ajay Kumar ${ }^{1} \quad$ Ujjwal Sonika ${ }^{1}$ Manish Gupta \\ Siddharth Srivastava ${ }^{1}$ Amol Dahale ${ }^{1}$ Ashok Dalal ${ }^{1, \odot}$
}

${ }^{1}$ Department of Gastroenterology, GB Pant Hospital, New Delhi,

Address for correspondence Ashok Dalal, MD, DM, Department of India Gastroenterology, Academic Block, GB Pant Hospital, 1, JLN Marg, New Delhi,India 110002,(e-mail: drashokdalal83@gmail.com).

Abstract
Keywords
- mobile phone
ingestion
- prisoners
- endoscopic removal of
mobile phone
- gastric foreign bodies
- unusual foreign bodies
ingestion

Objective Gastric foreign bodies are a common problem brought to an endoscopist. Prisoners are more prone to ingesting unusual foreign bodies for a secondary gain. The objective of this study was to study the profile of foreign body ingestion among the prisoners brought to a tertiary care center.

Methods This is a retrospective case series. All the prisoners brought for endoscopic removal of foreign bodies between January 2018 to December 2019 were included in the analysis. Patients' baseline characteristics, type of foreign body, management, and outcome were noted

Results A total of eight inmates presented with ingestion of foreign body to our department. Most common foreign bodies ingested were drug packets and mobile phones. All ingestions were for secondary gain. Endoscopic removal was successful in all cases.

Conclusion Endoscopic removal can be tried with all necessary precautions by an expert endoscopist.

\section{Introduction}

Gastric foreign body ingestion is a common emergency seen in gastroenterology. ${ }^{1}$ Accidental ingestion is most commonly seen in children. Although foreign body ingestion is rare in adults, it is more common in patients with psychiatric disorders. Intentional foreign body intake is seen commonly in prisoners. ${ }^{2}$ Several studies in various countries have evaluated the epidemiology of foreign body ingestion in prisoners. $^{2-4}$ In Ohio state in the US, the prevalence of intentional foreign body ingestion among prisoners was reported as 1 in 1900 inmates. $^{2}$

The foreign bodies ingested by prisoners are usually different from those in the general population. These may include objects which could provide them any secondary gain or come about on account of the psychiatric illness suffered by the prisoners. ${ }^{2}$ It can also be for unscrupulous transport of the objects in and out of prison.

There is no data from India regarding the spectrum of foreign body ingestion among prisoners. We aimed to study the profile of foreign body ingestion among the prisoners who consulted our department.

\section{Material and Methods}

We retrospectively reviewed the endoscopic records of the Department of Gastroenterology from the period of January 2018 to December 2019. The ethical clearance was waived-off by ethics committee in view of the retrospective nature of the study.

The prisoners with foreign body ingestion who underwent upper gastrointestinal (UGI) endoscopy were included published online April 19, 2021
DOI https://doi.org/ $10.1055 / \mathrm{s}-0041-1731963$ ISSN 0976-5042 (c) 2021. Society of Gastrointestinal Endoscopy of India.

This is an open access article published by Thieme under the terms of the Creative Commons Attribution-NonDerivative-NonCommercial-License, permitting copying and reproduction so long as the original work is given appropriate credit. Contents may not be used for commercial purposes, or adapted, remixed, transformed or built upon. (https://creativecommons.org/licenses/by-nc-nd/4.0/).

Thieme Medical and Scientific Publishers Pvt. Ltd. A-12, 2nd Floor, Sector 2, Noida-201301 UP, India 
in the study. The patient's baseline characteristics, clinical symptoms, type of foreign body, radiological investigations, and management were recorded. Qualitative data are represented as numbers (percentage). Quantitative data are represented as median and range.

\section{Management Protocol}

All the patients were evaluated in our outpatient clinic. Patients were evaluated clinically and an X-ray was done before endoscopy to confirm the location and types of foreign body in all the patients. The patients underwent UGI endoscopy after overnight fasting. All the endoscopies were performed by an expert endoscopist. During the procedure, foreign bodies were identified and were removed using appropriate accessories. A relook endoscopy was done after the removal of the foreign body to rule out any complications in the same sitting. Patients were observed for 6 hours postforeign body removal and then discharged.

\section{Results}

A total of 178 foreign bodies were removed in our department during the study period of 2 years. Eight inmates presented with ingestion of foreign body to our department in the study period. Foreign body removal in prisoners accounted for $4.49 \%$ of the total. All 8 (100\%) were males. The median age was 25 (range 22-35) years. Median number of ingested foreign bodies were 4 (range 1-10). None of the patients reported any symptoms due to foreign body ingestion. All the prisoners were brought by the jail authorities either after they detected the metallic foreign bodies on an X-ray or the inmate disclosed the history of foreign body ingestion.

The most common foreign bodies ingested were drug packets and mobile phones (-Table 1 ). All these foreign bodies were ingested for secondary gain as per the recorded history. The radio-opaque foreign bodies like mobile phones ( - Fig. 1) and surgical blades were visible on the X-ray. Drug packets were usually incidental findings on endoscopy or the prisoner himself gave the history of ingestion of these foreign bodies.

The exact time of ingestion was not exactly provided by the patients, but all of these inmates were in prison for more than a month. One inmate (S. No.8, - Table 1) who had ingested a mobile phone provided the history of ingesting the mobile phone a year back.

Mobile phones in most of these prisoners were neatly covered in a double sheath of polythene with methylene blue in between those two layers (-Fig. 2). The drug packets were also neatly packed in multiple layers of polythene to prevent rupture ( - Fig. 3). As per the recorded history, the purpose of methylene blue was to detect the rupture of polythene wrapping. One prisoner had ingested a surgical blade (with intact packaging) and the other had ingested a gold chain along with the drug packets.

These inmates gave the history of themselves being professional body packers but denied sharing the history of their previous ingestion. They used to vomit out these phones and drug packets after reaching jail with self-induced emesis after drinking large quantities of edible oils. There was no seepage detected onto the mobile phones and the battery was intact in all cases. Similarly, there was no rupture of drug packets during endoscopic removal.

All the patients underwent UGI endoscopy without any sedation. All eight patients had successful endoscopic removal of their foreign bodies (-Figs. 2, 3) without any complications. All the foreign bodies were removed easily except for mild difficulty in one prisoner who had ingested the mobile phone a year back and was not professional (S. No. 8, - Table 1). Mobile phones and drug packets were removed using snare ( - Fig. $\mathbf{3} \mathbf{A}, \mathbf{B}$ ). There was no rupture of drug packets during endoscopic removal. Postremoval relook endoscopy did not reveal any ulcer or fistula formation. A total of six mobile phones, nineteen drug packets, one gold chain, and one surgical blade were removed from these eight patients. After foreign body removal, all the prisoners were discharged and referred to psychiatrist for counselling.

Table 1 Baseline characteristics, profile of foreign body ingested, and endoscopic intervention in the patients

\begin{tabular}{|l|l|l|l|l|}
\hline S. no. & Age (years) & Gender & $\begin{array}{l}\text { Foreign body (number } \\
\text { ingested) }\end{array}$ & Endoscopic removal \\
\hline 1. & 27 & Male & $\begin{array}{l}\text { Mobile phone (1) } \\
\text { Drug packets (5) }\end{array}$ & Successful \\
\hline 2. & 25 & Male & Surgical blade (1) & Successful \\
\hline 3. & 27 & Male & $\begin{array}{l}\text { Mobile phone (3) } \\
\text { Drug packets (7) }\end{array}$ & Successful \\
\hline 4. & 22 & Male & Drug packets (5) & Successful \\
\hline 5 & 24 & Male & $\begin{array}{l}\text { Mobile phone (1) } \\
\text { Drug packets (3) }\end{array}$ & Successful \\
\hline 6 & 25 & Male & $\begin{array}{l}\text { Gold chain (1) } \\
\text { Drug packets (2) }\end{array}$ & Successful \\
\hline 7 & 28 & Male & Drug packets (2) & Successful \\
\hline 8 & 22 & Male & Mobile phone (1) & Successful \\
\hline
\end{tabular}




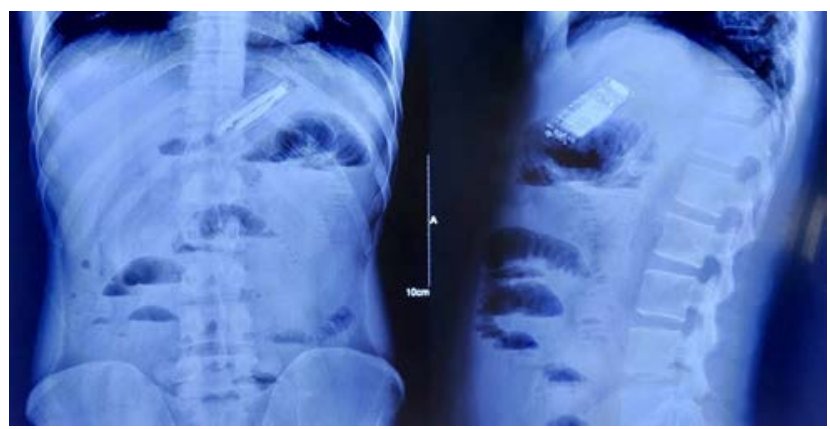

Fig. 1 X-ray abdomen showing foreign body, most likely a mobile phone.

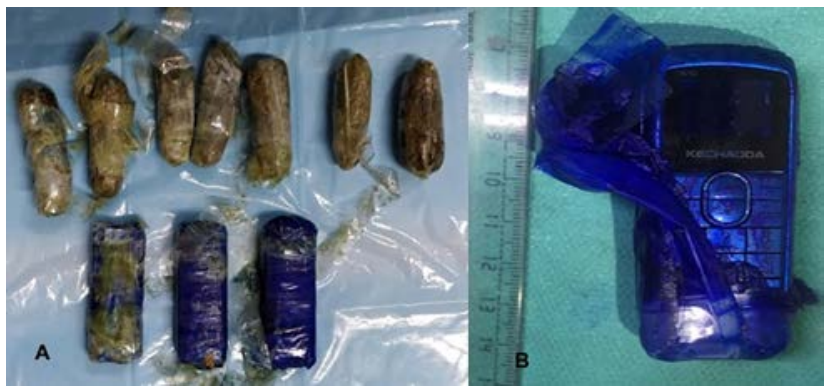

Fig. 2 (A) Mobile phone and drug packets removed. (B) Mobile phone showing wrapping in methylene blue covering.

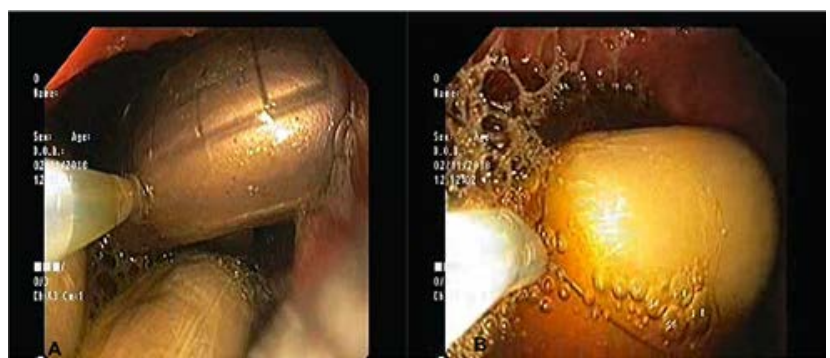

Fig. 3 Endoscopic removal of mobile phone (A) and drug packets (B) done with snare.

\section{Discussion}

The prisoners have a high frequency of intentional foreign body intake compared with the normal adult population. ${ }^{4}$ Our study had eight subjects included over 2 years.

The most common foreign bodies ingested were mobile phones and drug packets.

Mobile phone ingestion seems to be a recent phenomenon as technology has advanced, and the size of the basic mobile phone has shrunken, allowing it to be ingested and vomited in the prison. There are some previous case reports of mobile phone ingestion available..$^{5-7}$ Two of these case reports have shown successful endoscopic retrieval of these mobile phones, while the rest required surgery. ${ }^{5,6} \mathrm{We}$ have also reported the first case of this series (S No 1) as a case report. ${ }^{8}$ While comparing to studies from other countries, mobile phone ingestion is common in our setup. ${ }^{9-11}$ The prisoners used to vomit these drug packets rather than letting them pass out in the stool. It should be noted that these packets were intentionally made of relatively large size by the packers, so that they can stay in the stomach and can be vomited back.

These foreign bodies were carefully packed in strong plastics to prevent rupture of wrapping during emesis. This history gave us the confidence to try endoscopic removal in these cases. Endoscopic removal of foreign bodies was successful in all the cases, and there were no complications. Mobile phones were easily removed in most of these prisoners because of the lax lower and upper esophageal sphincters. Reason for laxity of sphincters probably was their habit of vomiting out of these objects in the past, as most of them were professional body packers. For drug packets ingestion, it is usually recommended to either wait for their spontaneous passage in stools or to go for surgery rather than endoscopic removal. ${ }^{12}$ Reasons for endoscopic removal of drug packets in our study were multifactorial, including ingestion of packets many months back, refusal to undergo surgery by prisoners and request by them for endoscopic removal, and finally packaging of drug packets in strong plastics to prevent rupture. Fortunately, all the drug packets were safely removed using a snare. Given the rarity of these interesting encounters, although of small size, this study gives a glimpse of the profile of foreign body ingestion among the prisoners in an Indian setup.

This study also demonstrates that endoscopic removal of mobile phone and drug packets is feasible and safe. Our study has the limitation of small sample size and retrospective nature.

\section{Conclusion}

To conclude, our study highlights that foreign body ingestion among prisoners may include unusual objects like mobile phones and drug packets, with secondary gain as the chief motive. Endoscopic removal is a safe and effective treatment in these subjects.

\section{Funding}

None.

\section{Conflict of Interest}

None.

\section{Acknowledgment}

None.

\section{References}

1 Birk M, Bauerfeind P, Deprez PH, et al. Removal of foreign bodies in the upper gastrointestinal tract in adults: European Society of Gastrointestinal Endoscopy (ESGE) Clinical Guideline. Endoscopy 2016;48(5):489-496

2 Dalal PP, Otey AJ, McGonagle EA, et al. Intentional foreign object ingestions: need for endoscopy and surgery. J Surg Res 2013;184(1):145-149

3 Bisharat M, O'Donnell ME, Gibson N, et al. Foreign body ingestion in prisoners - the Belfast experience. Ulster Med J 2008;77(2):110-114 
4 Evans DC, Wojda TR, Jones CD, Otey AJ, Stawicki SP. Intentional ingestions of foreign objects among prisoners: A review. World J Gastrointest Endosc 2015;7(3):162-168

5 Obinwa O, Cooper D, O'Riordan JM. An ingested mobile phone in the stomach may not be amenable to safe endoscopic removal using current therapeutic devices: a case report. Int J Surg Case Rep 2016;22:86-89

6 Qureshi NA, Cherian N, Ben-Hamida A, Solkar MH. Endoscopic retrieval of an intentionally ingested mobile phone in an adult: first case report of its kind. Ann Clin Case Rep 2016;1:1172

7 O'Connor PD, Sciarra J. Retrieval of an unusual foreign body. Cureus J Med Sci 2019;11(11):e6110

8 Pawar A, Dalal A, Dahale A, Sachdeva S, Puri AS. Mobile phone in stomach-Is endoscopic trial worthwhile?: a case report. J Dig Endosc 2020;11(2):165-167
9 Lee TH, Kang YW, Kim HJ, et al. Foreign objects in Korean prisoners. Korean J Intern Med (Korean Assoc Intern Med 2007;22(4):275-278

10 Volpi A, Laforgia R, Lozito C, et al. Ingestion of foreign bodies among prisoners: a ten years retrospective study at University Hospital of Southern Italy. G Chir 2017;38(2):80-83

11 Ribas Y, Ruiz-Luna D, Garrido M, Bargalló J, Campillo F. Ingested foreign bodies: do we need a specific approach when treating inmates? Am Surg 2014;80(2):131-137

12 Glovinski PV, Lauritsen ML, Bay-Nielsen M, Brandstrup B, Bisgaard T. Asymptomatic body packers should be treated conservatively. Dan Med J 2013;60(11):A4723 